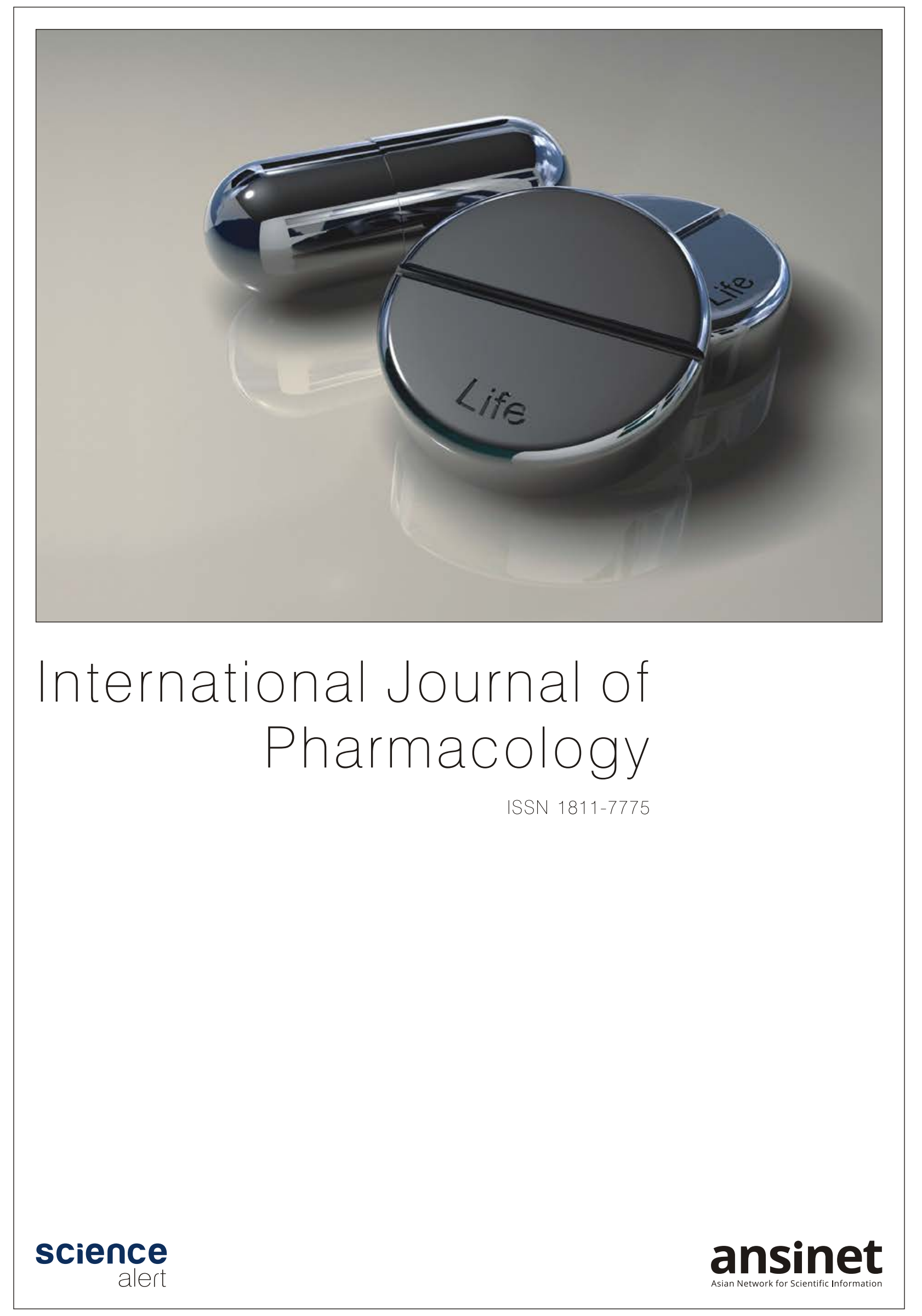




\title{
Ellagic Acid Loaded TPGS Micelles for Enhanced Anticancer Activities in Ovarian Cancer
}

\author{
${ }^{1}$ Mohammad Y. Alfaifi, ${ }^{1,2}$ SeragEldin I. Elbehairi, ${ }^{1}$ Ali A. Shati, ${ }^{3}$ Usama A. Fahmy, ${ }^{3}$ Nabil A. Alhakamy and \\ ${ }^{3}$ Shadab Md \\ ${ }^{1}$ Department of Biology, Faculty of science, King Khalid University, 9004 Abha, Saudi Arabia \\ ${ }^{2}$ Cell Culture Laboratory, Egyptian Organization for Biological Products and Vaccines, VACSERA Holding Company, 51 Wezaret El-Zeraa Street, \\ Agouza, Giza, Egypt \\ ${ }^{3}$ Department of Pharmaceutics, Faculty of Pharmacy, King Abdulaziz University, Jeddah, Saudi Arabia
}

\section{Abstract}

Background and Objective: Role of dietary phenolic compounds in the modification of pathophysiological conditions in cancer is immense. Thus, ellagic acid (EA), a natural polyphenolic compound has been recognized for its anticancer activity in different preclinical studies. However, clinical application is limited because of its poor aqueous solubility and thereby, inadequate oral bioavailability. The present work was aimed to formulate micellar delivery, an effective nano-delivery tool for EA, using D- $\alpha$-tocopheryl polyethylene glycol succinate (TPGS) by film-hydration method. Materials and Methods: Film hydration method was introduced to encapsulate EA within the TPGS micellar structure, which was then characterized and evaluated for in vitro release study. EA-TPGS micelle was further exposed to OVACR3 to determine cancer protecting potential of the formulation. Results: The delivery system (EA-TPGS micelles) consists of spherical shape of $113.2 \pm 23 \mathrm{~nm}$ with $0.260 \pm 0.038 \mathrm{PDI}$ and drug-encapsulation efficiency of $88.67 \% \pm 3.21$. In vitrorelease profile in the phosphate-buffer saline was found to observe sustained pattern with $67.8 \%$ cumulative release within $12 \mathrm{~h}$. Further, a dose dependent cytotoxicity of EA-TPGS micelles was observed on OVACR3 cells with an IC 50 value of $12.36 \mu \mathrm{M}$. EA-TPGS significantly reduced viable cells via arrest of $\mathrm{G} 1$ phase of cell cycle and thereby induce apoptosis probably through inhibiting p15 and p21. Decreased fluorescence unit in ROS determination assay also reflected potential antioxidant activity of the EA-TPGS. Conclusion: These findings strengthened that use of EA-TPGS micelles could overcome the limitations in delivery of hydrophobic chemotherapeutics through oral route.

Key words: Ellagic acid, micelles, apoptosis, ovarian cancer, cell cycle, chemotherapeutic agents, cytotoxicity, anti-cancer activity

Citation: Mohammad Y. Alfaifi, SeragEldin I. Elbehairi, Ali A. Shati, Usama A. Fahmy, Nabil A. Alhakamy and Shadab Md, 2020. Ellagic acid loaded TPGS micelles for enhanced anticancer activities in ovarian cancer. Int. J. Pharmacol., 16: 63-71.

Corresponding Authors: Shadab Md, Department of Pharmaceutics, Faculty of Pharmacy, King Abdulaziz University, 21589 Jeddah, Saudi Arabia Mohammad Y. Alfaifi, Department of Biology, Faculty of science, King Khalid University, 9004 Abha, Saudi Arabia

Copyright: @ 2020 Mohammad Y. Alfaifi et al. This is an open access article distributed under the terms of the creative commons attribution License, which permits unrestricted use, distribution and reproduction in any medium, provided the original author and source are credited.

Competing Interest: The authors have declared that no competing interest exists. 


\section{INTRODUCTION}

World Health Organization (WHO) has recognized cancer as the most critical health hazard with leading cause of death, where about $70 \%$ of deaths are resulted in low-and/or middle-income countries ${ }^{1}$. Among these, ovarian cancer is the most deadly gynecologic cancer and usually diagnosed at the late stage of the disease ${ }^{2}$. Recent treatment for this fatal disease includes paclitaxel and platinum based treatments and their combinations, where a significant number of patients develop resistance to these drugs due to their unpredictable time of response ${ }^{3}$. Thus, recent researches focus on natural compounds for their overwhelming outcomes in several diseases, such as diabetes ${ }^{4}$, cognitive disorder ${ }^{5}$, cardiac complications $^{6}$ and also in cancer, which have shown to double the life-span of the patients in this century ${ }^{7}$. Presence of secondary metabolites as bioactive compounds, including polyphenols, in these plant resources known to responsible for their potential role. Ellagic acid (EA), a natural polyphenol, is a dimeric form of gallic acid. Different natural sources of EA include grapes, pomegranates, strawberries, raspberries, blueberries, walnuts etc ${ }^{8}$. A wide varieties of potential biological role has been observed with EA in different pre-clinical studies, such as cardiac ailments, cancer and other medical issues ${ }^{9}$, however, its' poor aqueous solubility and low bioavailability limits its therapeutic application in human. Improvement of solubility and thereby bioavailability of poorly soluble drugs are circumvented by the nanotechnological deliveries ${ }^{10}$. Amphiphilic spherical nanocarriers, such as micelles of poorly soluble drugs possess superior property to incorporate hydrophobic agents within the hydrophobic core, where the hydrophilic shells of the micelle helps to stabilize in aqueous environment ${ }^{11}$. This unique structure of the formulation leads towards improvement of pharmacokinetics and pharmacodynamic properties through escape of reticuloendothelial system due to its nanometric size range $(<200 \mathrm{~nm})$. Therefore, increased circulation of these nanocarriers lead to increased accumulation within the cancerous environment through the enhanced permeability and retention effect (EPR effect), help in delivery of the entrapped drug to the cancer cells ${ }^{12}$. Further, use of D- $\alpha$-tocopheryl polyethylene glycol 1000 succinate (i.e., TPGS), an amphiphilic copolymer has gained recent attention in the development of novel pharmaceutical formulations due to their biocompatibility, which is also recognized by the Food and Drug Administration (FDA) as a pharmaceutical ingredient ${ }^{13-15}$. Existence of polyethylene glycol (PEG) in this amphiphilic non-ionic macromolecule further help in the improvement of EPR for prolonged circulation of nanocarrier, thus enhance bioavailability ${ }^{16}$.
The micellar deliveries using TPGS are also found to be a P-glycoprotein (Pgp) inhibitor, a multidrug resistant protein known to efflux out the chemotherapeutics from the cancer cells $^{17}$. Thus, the micellar delivery could improve the pharmacokinetic profile of the formulation. Therefore, in order to obtain the physiological effect of ellagic acid and TPGS in a single formulation, the objective of the present study was planned. The present study was aimed to develop a TPGS based micellar delivery system of EA by using thin-film hydration technique to target ovarian cancer. EA-TPGS micelles were characterized for their size, surface morphology, entrapment efficiency and release pattern. Further, activity of the micelles was verified in vitro in ovarian cancer cell lines for cytotoxicity and apoptotic potential.

\section{MATERIALS AND METHODS}

The active ingredient, ellagic acid (EA), d- $\alpha$-tocopheryl polyethylene glycol 1000 succinate (TPGS), 2,7 dichlorofluorescein diacetate and dialysis membrane of 12 kDa were purchased Sigma Aldrich, USA. OVCAR3 (epithelial ovarian cancer cell lines) was obtained from ATCC (King Fahd medical centre, Jeddah, Saudi Arabia) and maintained in mammalian cell culture media (RPMI 1640, Life Technologies (Carlsbad, CA) which was supplemented with $10 \mathrm{U} \mathrm{mL}^{-1}$ penicillin and $10 \mu \mathrm{g} \mathrm{mL}^{-1}$ streptomycin, $10 \%(\mathrm{v} / \mathrm{v})$ fetal bovine serum and $0.25 \mu \mathrm{g} \mathrm{mL}^{-1}$ of amphotericin $B$ in a humidified environment of $37^{\circ} \mathrm{C}, 5 \% \mathrm{CO}_{2}$. Rests of the chemicals used in this current experiment were of analytical grade. This entire study was carried out at the faculty of Pharmacy, King Abdul-Aziz university, Jeddah, Saudi Arabia in the period from May-October, 2019. Thereafter, the obtained data were compiled to bring up into this manuscript.

Preparation of EA-TPGS- micelles: EA loaded TPGS micelles were prepared by thin film hydration method ${ }^{18}$ with minor modification. Firstly, EA (50 mg) and TPGS $(300,500,700 \mathrm{mg})$ were dissolved in ethanol via constant stirring (magnetic stirrer) for $5 \mathrm{~min}$. Subsequently, the ethanol was evaporated from dispersion by a rotary evaporator (BÜCHI Labortechnik $A G$, Flawil, Switzerland) which lead to formation of TPGS thin film dispersed with EA. Micelles were formed by hydration of TPGS thin film with water with agitation. The resultant micelles were then centrifuged at 30,000 rpm for $45 \mathrm{~min}$ at $4^{\circ} \mathrm{C}$ followed by filtration of supernatant using cellulose acetate membrane filter. The residue was lyophilized using a freeze dryer (alpha 1-2 LD plus lyophilizer, Christ, Osterode am Harz, Germany) for $48 \mathrm{~h}$ and stored for further characterization. 
Characterization of EA loaded (EA-TPGS) micelles Mean particle size, polydispersity index (PDI) and surface morphology of the micelle: Mean particle size and PDI of EA-TPGS micelles were investigated by light scattering technique using a Zetatrac particle size analyzer (Microtrac Inc., Montgomeryville, PA, USA). The surface morphology and shape was examined by transmission electron microscopy (TEM) system (100CX, JEOL, Tokyo, Japan). A sample of diluted EA-TPGS micelles were prepared by placing a drop of micelles onto a microscope grid and dried on the grid after staining with $2 \%$ uranyl acid ${ }^{19}$.

Encapsulation efficiency: Encapsulation efficiency ofEA-TPGS micelles were examined by modified reversed-phase HPLC method. Briefly, micelles dispersion was dissolved in ethanol with subsequent filtration to remove undissolved polymeric content. After dilution with appropriate mobile phase (water:methanol:acetonitrile:orthophosphoric acid (60:30:10:1\%) used as a mobile phase. The flow rate was


$10 \mu \mathrm{L}$ of sample was injected into HPLC column $(250 \times 4.6 \mathrm{~mm}$ i.d. Symmetry-C18, $5 \mu \mathrm{m}$ column)and UV detection was executed at $\lambda_{\max }=262 \mathrm{~nm}$. Following formula was used to determine the encapsulation efficiency of EA-TPGS micelles ${ }^{19}$ :

$$
\begin{aligned}
& \text { Encapsulation } \\
& \text { efficiency }(\%)
\end{aligned}=\frac{\text { Weight of the drug in EA - TPGS - M }}{\text { Weight of the feeding drug }} \times 100
$$

In vitro drug release of EA-TPGS micelles: Time dependent release of EA from micelles were evaluated by dialysis bag method in which EA-TPGS micelles (equivalent to $5 \mathrm{mg}$ of EA) were placed in dialysis bag (12 kDa cut off) and closed from both the ends ${ }^{20}$. This dialysis bag was submerged into phosphate buffer saline $(\mathrm{pH}$ 7.4) with stirring at $100 \mathrm{rpm}$ at $37^{\circ} \mathrm{C}$. Afterward, $10 \mathrm{~mL}$ of sample were withdrawn at definite time interval and released drug content was estimated by HPLC as discussed earlier.

Cytotoxicity assay: Cytotoxicity of EA-TPGS and EA was evaluated by MTT assay ${ }^{21}$.OVCAR3 cells were seeded the density of $5 \times 10^{3}$ cells onto each well of 96-well plates and incubate for 24-48 h. After incubation cells were treated with various concentration of free EA and EA-TPGS micelles for $24 \mathrm{~h}$. Subsequently, free drug and drug loaded micelles were removed and treated cells were incubated with $M T T$ solution $(0.5 \% \mathrm{w} / \mathrm{v})$ for $4 \mathrm{~h}$. Finally, after the removal of MTT solution cells were exposed to $150 \mu \mathrm{L}$ DMSO for the removal of formazan crystals. Number of living cells were calculated based on optical density of formazan at $490 \mathrm{~nm}$ using ELISA microplate reader.
Cell cycle analysis: Determination of cell cycle distribution was estimated by the treatment of free EA and EA-TPGS micelles in OVCAR3 cells lines for a period of $12 \mathrm{~h}$ following the method depicted by Ling et a/.22. Following this, the cells were harvested and $75 \%$ ethanol was used to fix it at $20^{\circ} \mathrm{C}$ overnight. At that point, the cells were incubated with $5 \mu \mathrm{g} \mathrm{mL} \mathrm{m}^{-1}$ propidium iodide and $5 \mu \mathrm{g} \mathrm{mL}^{-1}$ of RNase for $3 \mathrm{~h}$ at room temperature in the dark. Subsequently, the number of cells at different stages of cell cycle were analysed by using flow cytometer (FACSCalibur, BD Bioscience). Data of 10,000 cells were composed for each data file, where the obtained data were analysed by using Multicycle software (Phoenix Flow Systems, San Diego, CA).

Apoptosis assay: Double staining technique were used to evaluated the apoptotic effect of free EA and EA-TPGS micelles on OVACR3 cells. Apoptosis of cells were assessed by an annexin V-fluorescein isothiocyanate (FITC) conjugate and propidium iodide $(\mathrm{PI})^{23}$. Firstly, the OVACR3 cells were exposed to EA and EA-TPGS containing media for $24 \mathrm{~h}$. Subsequently the cells were washed with phosphate buffer to remove free drug with micelles and processed for assay according to instruction of apoptosis detection kit (BD Bioscience, USA). Flow cytometer (FACSCalibur, BD Bioscience) were used to analyse the sample and obtained data were evaluated via Multicycle software (Phoenix Flow Systems, San Diego, CA).

Caspase-3 activity: The cells ( $5 \times 10^{3}$ cells) were seeded in a 96-well plate. After $24 \mathrm{~h}$ control, EA-TPGS micelles, TPGS and EA were put into the wells to induce apoptosis ${ }^{23}$. Caspase-3 activity was observed as per manufacturer instructions (USCN Life Science Inc., China).

Reactive oxygen species activity (ROS): Determination of the amount of ROS at intracellular compartment in treated cancer cells (OVCAR3) is determined by using 2,7 dichlorofluorescein diacetate ${ }^{24}$. ROS concentration in OVCAR3 cancer cells was measured, where $20 \times 10^{3}$ cells were seeded in 96 well plate and incubated for $24 \mathrm{~h}$ with $10 \mu \mathrm{mol} \mathrm{L}^{-1} 2,7$ dichlorofluorescein diacetate following washing in phosphate buffer saline. Subsequently, treated with free drug, TPGS, EA-TPGS and ROS levels were measured by flow cytometry following incubation with polyphenols for a 20 min duration.

Statistical analysis: Statistical analysis was performed by Student's t-test between two groups and one-way ANOVA for multiple groups. All results are given as Mean $\pm S D$. Differences are considered statistically significant at a level of $p<0.05$. 


\section{RESULT AND DISCUSSION}

\section{Mean particle size, polydispersity index (PDI) and surface} morphology of the micelle: The mean particle size and PDI of EA-TPGS micelles prepared by thin film hydration method was shown in Table 1. However, no significant effect was observed in particle size and PDI after increment in TPGS concentration. The particle size obtained by light scattering method (Fig. 1) was larger as compared to size obtained by TEM images (Fig. 2) could be due to hydration of particles. TEM images illustrate that drug loaded micelles exhibit spherical shape with uniform particle size. It is was evident from the literature that nano-carriers less than $200 \mathrm{~nm}$ can avoid reticuloendothelial system (RES) uptake along with the ease of extravasation ${ }^{17,25}$.
Encapsulation efficiencies: Encapsulation efficiency of EA-TPGS micelles was found to be $88.67 \pm 3.21 \%$ for F2 formulation, which shows significant increment in entrapment of EA from F1 formulation, however, no difference in drug entrapment was observed between F2 and F3. It is evident from the data that entrapment of EA in TPGS micelles was high due to solubility enhancement properties of TPGS due to its amphiphilic property contains polar terminal end with long hydrophobic carbon chain ${ }^{15,26}$. Similar trend was observed by Patra et al.27 when they compared the drug loading capacity of micelles with and without TPGS. They also exemplified the 1000 fold increase in solubility of hydrophobic drug in TPGS micelles compared to pure drug.

Table 1: Formulas of micelle formulations with different concentrations of TPGS and particle size, poly dispersity index and encapsulation efficiency of TPGS micelles

\begin{tabular}{|c|c|c|c|c|c|}
\hline Formulation & EA content (mg) & $\begin{array}{l}\text { TPGS concentration } \\
\left(\mathrm{mg} \mathrm{mL}^{-1}\right)\end{array}$ & Particle size $(\mathrm{nm})$ & Polydispersity index & $\begin{array}{l}\text { Encapsulation } \\
\text { efficiency (\%) }\end{array}$ \\
\hline EA-TPGS-F1 & 50 & 300 & $93.4 \pm 7.2$ & $0.311 \pm 0.021$ & $76.23 \pm 1.75$ \\
\hline EA-TPGS-F2 & 50 & 500 & $113.2 \pm 23$ & $0.260 \pm 0.038$ & $88.67 \pm 3.21$ \\
\hline EA-TPGS-F3 & 50 & 700 & $121.2 \pm 11.2$ & $0.282 \pm 0.012$ & $91.12 \pm 0.89$ \\
\hline
\end{tabular}

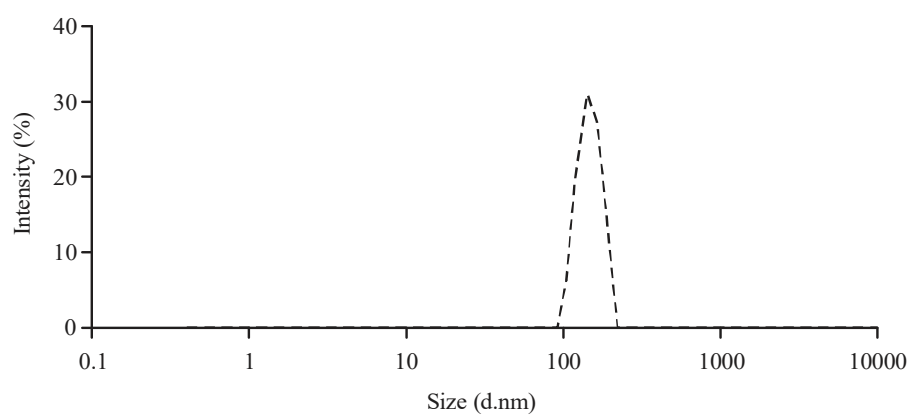

Fig. 1: Particle size and particle size distribution of EA- EA-TPGS micelles as measured by dynamic light scattering method



Fig. 2: TEM image of the formulation EA-TPGS micelles 


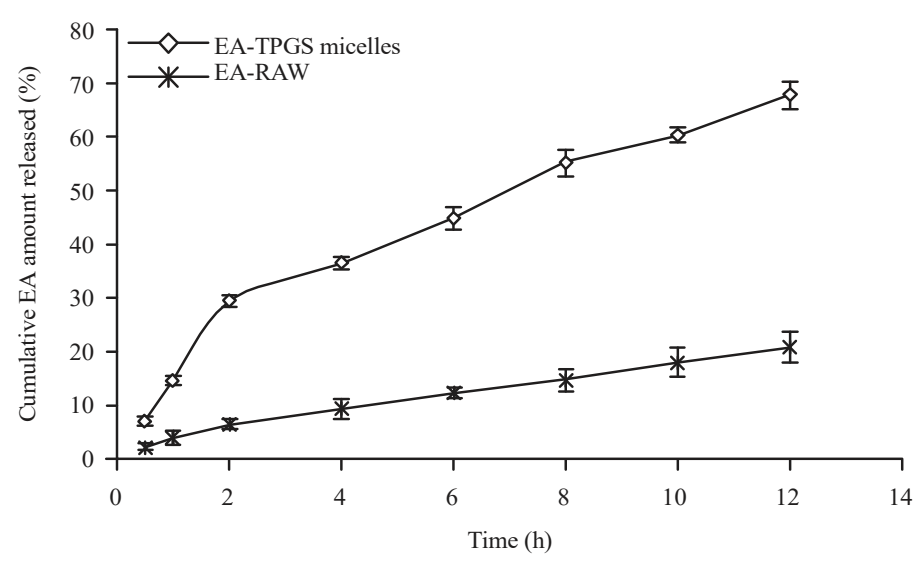

Fig. 3: In vitro release profiles of EA-TPGS micelles and EA suspension in phosphate buffer saline $(\mathrm{pH} 7.4)$

In vitro release study: In vitro release of EA from EA-TPGS-F2 was evaluated through dialysis bag method using phosphate buffer saline $(\mathrm{pH}$ 7.4) as dissolution media. The percentage release of Pure EA and EA-TPGS micelle is shown in Fig. 3. It is evident from the graph that release of EA from TPGS micelles (73.25\%) was approximately 3 fold higher than the suspension of the EA (20.2\%). This might be attributed to the transportation of EA loaded nanosize micelles through the pores of dialysis membrane. Release of free drug was only $20.2 \%$ after $12 \mathrm{~h}$, which denote to low solubility of parent drug in dissolution media. Therefore, the findings revealed the concept of nanocarrier help to improve release characteristics of the poorly water soluble drugs s,2, $^{29}$.

Cytotoxicity study: In vitro cytotoxicity test is the first step to evaluate the biocompatibility of polymer as well as affectivity of formulation on cancer cells. Cytotoxicity of micelles was evaluated by direct contact methods with cells as recommended by ISO guidelines on OVCAR3, which involves the placement of the test material directly on the cell culture medium. OVCAR3 cells were treated with pure drug (EA), TPGS and EA-TPGS-F2 and cell proliferation was observed as end point and results revealed the EA-TPGS-F2 is most efficacious in the inhibition of cancer cells in dose dependent manner (data not shown). IC50 value of EA-TPGS micelles, pure drug (EA) and TPGS were calculated from the data and recorded as $12.3,19.13$ and $113.9 \mu \mathrm{M}$, respectively. This depicted the cyto-compatibility of TPGS and cytotoxic nature of drug in free and entrapped form.

Cell cycle analysis: Cell cycle phase was analyzed by flow-cytometric determination of DNA content in OVCAR3 cells treated with free EA and EA-TPGS micelles. The data in Fig. 4a-d clearly showed there was rapid growth/cell proliferation for untreated OVCAR3 cells (control group) at G0/G1 phase $(54.41 \pm 1.99 \%)$, G2-M phase $(13.45 \pm 1.06 \%)$, $S$ phase $(36.65 \pm 2.13 \%)$ and pre G1 phase $(2.15 \pm 0.8 \%)$. The accumulation of cells in the pre-G phase was increased for free EA (12.45 $\pm 0.29 \%)$ and EA-TPGS micelles $(24.11 \pm 1.56 \%)$ as compared to the control group ( $2.15 \pm 0.58 \%)$ indicating that cells undergoing apoptosis. There was significant decrease in cell proliferation for both EA and EA-TPGS micelles compared to control group by increased number of cell accumulation at G2/M phase $(p<0.01)$. However the cell accumulation at G2/M phase was significantly higher with EA-TPGS micelles $(42.9 \pm 1.96 \%)$ compared to free EA solution (29.04 $\pm 2.09 \%)$ $(p<0.05)$ (Fig. 4d). Thus, it could be assumed that the increased arrest in G2/M phase of cell proliferation by EA-TPGS micelles might be due to synergistic effect of micellar delivery to increase cellular concentration of the drug and presence of TPGS. There was decrease in number of cell population on G1 phases for EA-TPGS micelles (32.77 $\pm 2.44 \%$ ) as compared to EA solution (47.5 $\pm 1.95 \%)$ and control group $(54.41 \pm 1.99 \%)$ (Fig. 4d). Cellular proliferation is imperative in regulation of the cell cycle, where the cell cycle abnormality is leading to cancer $^{30}$. G1 phase alteration is mainly due to cyclins, cyclin-dependent kinases and inhibitors of cyclin-dependent kinases. Cancer usually caused by dysregulation of inhibitors of cyclin-dependent kinases and two members ${ }^{31}$, p15 and p21. EA is reported to decrease expression of P53/P12 through inhibition of $\mathrm{G} 1$ phase $\mathrm{e}^{32}$. The present study findings are also in line to the reported results.

Determination of apoptotic potential: Similar results were observed from apoptotic assay, which showed marked increase in cell death in time dependent manner of 
Int. J. Pharmacol., 16 (1): 63-71, 2020


Fig. 4(a-d): Flow cytometric analysis showing the effect of (a) Control group, (b) Ellagic acid solution, (c) EA-TPGS micelles in OVCAR3 cancer cell at $24 \mathrm{~h}$ for cell cycle phases, the orange peak represent $\mathrm{G} 1$ phase in the cell cycle and the red peak represent G2/M phase in the cell cycle and (d) Represent bar graph of flow cytometry data for Pre G phase, G1 phase, $\mathrm{G} 2 / \mathrm{M}$ and $\mathrm{S}$ phase

Results are represented as the mean \pm SD of 3 distinct findings, $\mathrm{Y}$ axis represent number of cell population (\%), X-axis represent FL2H: Flow cytometric analysis stained with propidium iodide (PI) histograms, there was significant decrease in cell proliferation for both EA and EA-TPGS micelles compared to control group by increased number of cell accumulation at $\mathrm{G} 2 / \mathrm{M}$ phase $(\mathrm{p}<0.01)$

incubation. It was evident from Fig. 5, in the absence of EA (control group) majority of cells were viable, although after treating the cells with free EA there was an increase in early and late apoptosis. Similarly, when cells were treated with EA-TPGS micelles, there was a significant increase in early apoptosis cells accompanied by higher percentage increase in late apoptosis cells. The total apoptosis of cancer cell was higher in EA-TPGS-F2 (24\%) and free drug (12\%) incubated cell compared to non-treated cells (2\%).

This may attribute to loss in cell membrane integrity and caspase 3 activation ${ }^{33}$ as caspase- 3 production increase from 33.2-373.6 $\mathrm{pg} \mathrm{mL}^{-1}$ in control cells after incubating with EA-TPGS. Similarly, cell exposed to free drug also resulted in higher concentration (223 pg mL ${ }^{-1}$ ) of caspase-3 (Fig. 6a). Therefore, higher apoptotic potential of the miceller delivery showed to produce higher necrosis to the cellular growth (2.61\%) compared to $1.79 \%$ in free drug group (Fig. 4). As evident from the literature, caspases are crucial mediator for apoptosis by cleaving many key cellular protein. It is also associated with the formation with apoptotic bodies and dismantling of cells ${ }^{34}$.

Measurement of ROS: Malignant transformation of normal cells to cancer showed higher ROS level to maintain tumor progression and oncogenic phenotype. In this study level of ROS was determined by intensity of fluorescence, which is proportional to intracellular ROS by the oxidant-sensing probe 2,7-dichlorofluorescein diacetate due to hydrolysis on reaction with ROS. Figure $6 \mathrm{~b}$ illustrated that EA-TPGS showed significant decrease in ROS level (fluorescence intensity) as 

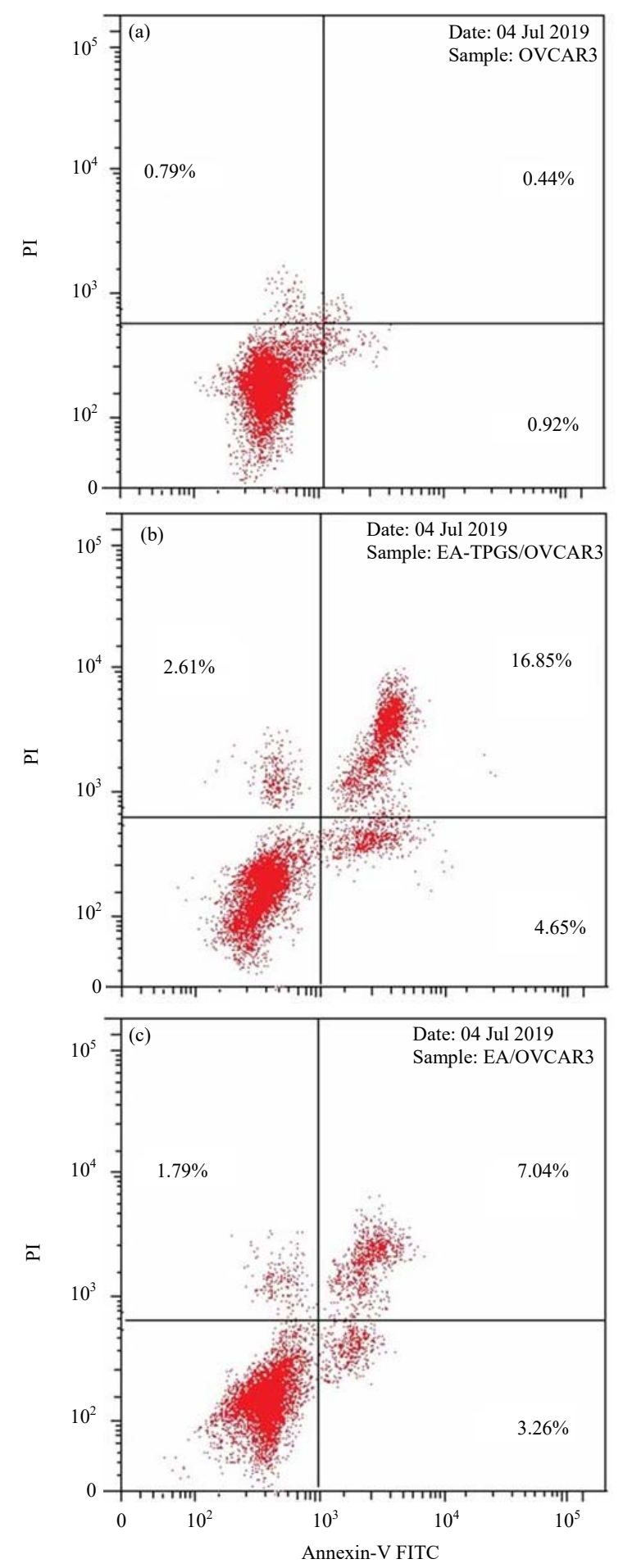

Fig. 5(a-c): Apoptotic characteristics of incubated OVCAR3 cancer cells (a) Control, (b) EA-TPGS micelles and (c) Free drug (EA)

compared EA and TPGS $(p \leq 0.05)$. However, there was no significant differences in between EA-TPGS micelles and control group ( $p \geq 0.05$ ), when compared to control. This may
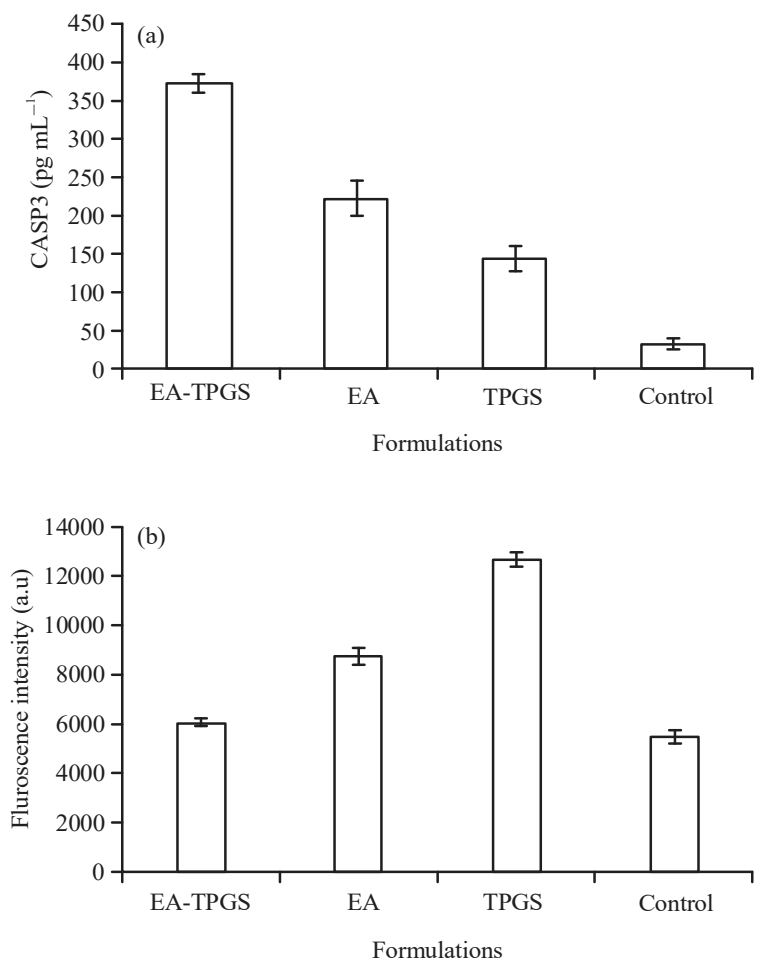

Fig. 6(a-b): (a) Caspase 3 activity and (b) ROS measurement of incubated OVCAR3 cancer cells on control, EA-TPGS micelles, free drug (EA) and TPGS

be attributed to ability of EA to scavenge ROS. In contrast, TPGS have higher fluorescence then free $E A$, which denote the intrinsic anticancer activity of TPGS by inhibiting cell growth via generation of $\operatorname{ROS}^{35}$, where a synergy is observed in EA-TPGS.

\section{CONCLUSION}

It could be concluded that the nano-sized and smooth surfaced micelles were formed by film hydration method with high drug entrapment. In vitrorelease profile revealed that 5 -fold higher release of the drug from micelles compared to free drug in sustained manner. Additionally, micelles entrapped EA showed highest cytotoxicity compared to free drug and TPGS in dose dependent manner. Further, suppression of G1/M phase by the EA-TPGS-F2 could be due to suppression of p15 and p21 promoter genes within the cancer cell with an improved apoptotic potential along with increased antioxidant potential of the formulation. These finding demonstrated the potential of EA-TPGS micelles in the treatment of ovarian cancer. 


\section{SIGNIFICANT STATEMENT}

This study represented the development and characterization of ellagic acid loaded TPGS-polymeric micelles that can be beneficial for effective delivery of ellagic acid using oral routes of administration. This novel delivery system of ellagic acid was shown to have promising outcome against ovarian cancer cells. This study will help the researcher to uncover critical ideas on ellagic acid against cancer treatment as bioavailability problem could be solved using micellar delivery system of the drug and simultaneously to focus towards using of this delivery tool for delivery of hydrophobic therapeutics via oral route that many researchers are still anxious to explore.

\section{ACKNOWLEDGMENT}

The authors extend their appreciation to the Deanship of Scientific Research at King Khalid University for funding this work through Research Group project under grant number (R.G.P.I/149/40).

\section{REFERENCES}

1. WHO., 2018. Cancer. World Health Organization, Geneva, Switzerland, September 12, 2018. https://www.who.int/en /news-room/fact-sheets/detail/cancer

2. Marcus, C.S., G.L. Maxwell, K.M. Darcy, C.A. Hamilton and W.P. McGuire, 2014. Current approaches and challenges in managing and monitoring treatment response in ovarian cancer. J. Cancer, 5: 25-30.

3. Salzano, G., G. Navarro, M.S. Trivedi, G. De Rosa and V.P. Torchilin, 2015. Multifunctional polymeric micelles co-loaded with anti-survivin sirna and paclitaxel overcome drug resistance in an animal model of ovarian cancer. Mol. Cancer Ther., 14: 1075-1084.

4. Kesharwani, P., B. Gorain, S.Y. Low, S.A. Tan and E.C.S. Ling et al., 2018. Nanotechnology based approaches for anti-diabetic drugs delivery. Diabetes Res. Clin. Pract., 136: 52-77.

5. Gorain, B., H. Choudhury, M. Pandey, H.L.B. Mohd Zaki and N.I.B. Bakar et al., 2018. Mechanistic description of natural herbs in the treatment of dementia: A systematic review. Curr. Psychopharmacol., 7: 149-164.

6. Chen, X., J. Qian, L. Wang, J. Li and Y. Zhao et al., 2018. Kaempferol attenuates hyperglycemia-induced cardiac injuries by inhibiting inflammatory responses and oxidative stress. Endocrine, 60: 83-94.

7. Demain, A.L. and P. Vaishnav, 2011. Natural products for cancer chemotherapy. Microb. Biotechnol., 4: 687-699.
8. Kim, S., M.W. Gaber, J.A. Zawaski, F. Zhang, M. Richardson, X.A. Zhang and Y. Yang, 2009. The inhibition of glioma growth in vitro and in vivo by a chitosan/ellagic acid composite biomaterial. Biomaterials, 30: 4743-4751.

9. Arulmozhi, V., K. Pandian and S. Mirunalini, 2013. Ellagic acid encapsulated chitosan nanoparticles for drug delivery system in human oral cancer cell line (KB). Colloids Surf. B: Biointerf., 110: 313-320.

10. Choudhury, H., M. Pandey, T.H. Yin, T. Kaur and G.W. Jia et al., 2019. Rising horizon in circumventing multidrug resistance in chemotherapy with nanotechnology. Mater. Sci. Eng.: C, 101: 596-613.

11. Brinkman, A.M., G. Chen, Y. Wang, C.J. Hedman and N.M. Sherer et al., 2016. Aminoflavone-loaded EGFR-targeted unimolecular micelle nanoparticles exhibit anti-cancer effects in triple negative breast cancer. Biomaterials, 101: 20-31.

12. Movassaghian, S., O.M. Merkel and V.P. Torchilin, 2015. Applications of polymer micelles for imaging and drug delivery. Wiley Interdiscip. Rev.: Nanomed. Nanobiotechnol., 7: 691-707.

13. Wang, Y., Y. Ding, J. Zhao, C. Wang and M. Gao et al., 2019. Dihydroartemisinin and doxorubicin co-loaded Soluplus ${ }^{\circledR}$ TPGS mixed micelles: Formulation characterization, cellular uptake and pharmacodynamic studies. Pharm. Dev. Technol., 24: 1125-1132.

14. Fan, Z., C. Chen, X. Pang, Z. Yu and Y. Qi et al., 2015. Adding vitamin E-TPGS to the formulation of genexol-PM: Specially mixed micelles improve drug-loading ability and cytotoxicity against multidrug-resistant tumors significantly. PLoS One., Vol. 10, No. 5. 10.1371/journal.pone.0120129.

15. Choudhury, H., B. Gorain, M. Pandey, S.A. Kumbhar, R.K. Tekade, A.K. Iyer and P. Kesharwani, 2017. Recent advances in TPGS-based nanoparticles of docetaxel for improved chemotherapy. Int. J. Pharm., 529: 506-522.

16. Feng, S.S., 2006. New-concept chemotherapy by nanoparticles of biodegradable polymers: Where are we now? Nanomedicine, 1: 297-309.

17. Choudhury, H., B. Gorain, M. Pandey, R. Kaur and P. Kesharwani, 2019. Strategizing biodegradable polymeric nanoparticles to cross the biological barriers for cancer targeting. Int. J. Pharm., 565: 509-522.

18. Ai, X., L. Zhong, H. Niu and Z. He, 2014. Thin-film hydration preparation method and stability test of DOX-loaded disulfide-linked polyethylene glycol 5000-lysine-ditocopherol succinate nanomicelles. Asian J. Pharm. Sci., 9: 244-250.

19. Wang, Y., X. Ke, Z.X. Voo, S.S.L. Yap and C. Yang et al., 2016. Biodegradable functional polycarbonate micelles for controlled release of amphotericin B. Acta Biomater., 46: $211-220$. 
20. Gorain, B., H. Choudhury, A. Kundu, L. Sarkar, S. Karmakar, P. Jaisankar and T.K. Pal, 2014. Nanoemulsion strategy for olmesartan medoxomil improves oral absorption and extended antihypertensive activity in hypertensive rats. Colloids Surf. B: Biointerf., 115: 286-294.

21. Bahuguna, A., I. Khan, V.K. Bajpai and S.C. Kang, 2017. MTT assay to evaluate the cytotoxic potential of a drug. Bangladesh J. Pharmacol., 12: 115-118.

22. Ling, Y.H., L. Liebes, J.D. Jiang, J.F. Holland and P.J. Elliott et al., 2003. Mechanisms of proteasome inhibitor PS-341-induced $\mathrm{G}_{2}-\mathrm{M}$-phase arrest and apoptosis in human non-small cell lung cancer cell lines. Clin. Cancer Res., 9: 1145-1154.

23. Shimbo, K., G.W. Hsu, H. Nguyen, S. Mahrus, J.C. Trinidad, A.L. Burlingame and J.A. Wells, 2012. Quantitative profiling of caspase-cleaved substrates reveals different drug-induced and cell-type patterns in apoptosis. Proc. Natl. Acad. Sci. USA., 109: 12432-12437.

24. Wang, H. and J.A. Joseph, 1999. Quantifying cellular oxidative stress by dichlorofluorescein assay using microplate reader. Free Rad. Biol. Med., 27: 612-616.

25. Muthu, M.S., R.V. Kutty, Z. Luo, J. Xie and S.S. Feng, 2015. Theranostic vitamin E TPGS micelles of transferrin conjugation for targeted co-delivery of docetaxel and ultra bright gold nanoclusters. Biomaterials, 39: 234-248.

26. Gorain, B., H. Choudhury, M. Pandey and P. Kesharwani, 2018. Paclitaxel loaded vitamin E-TPGS nanoparticles for cancer therapy. Mater. Sci. Eng.: C, 91: 868-880.

27. Patra, A., S. Satpathy, A. Shenoy, J. Bush, M. Kazi and M.D. Hussain, 2018. Formulation and evaluation of mixed polymeric micelles of quercetin for treatment of breast, ovarian and multidrug resistant cancers. Int. J. Nanomed., 13: $2869-2881$
28. Gao, F., Z. Zhang, H. Bu, Y. Huang and Z. Gao et al., 2011. Nanoemulsion improves the oral absorption of candesartan cilexetil in rats: Performance and mechanism. J. Control. Release, 149: 168-174.

29. Choudhury, H., B. Gorain, S. Karmakar, E. Biswas and G. Dey et al., 2014. Improvement of cellular uptake, in vitro antitumor activity and sustained release profile with increased bioavailability from a nanoemulsion platform. Int. J. Pharm., 460: 131-143.

30. Massagué, J., 2004. G1 cell-cycle control and cancer. Nature, 432: 298-306.

31. Chen, W., M. Huang, D. Sun, R. Kong and T. Xu et al., 2016. Long intergenic non-coding RNA 00152 promotes tumor cell cycle progression by binding to $\mathrm{EZH} 2$ and repressing p15 and p21 in gastric cancer. Oncotarget, 7: 9773-9787.

32. Narayanan, B.A., O. Geoffroy, M.C. Willingham, G.G. Re and D.W. Nixon, 1999. p53/p21 (WAF1/CIP1) expression and its possible role in $\mathrm{G} 1$ arrest and apoptosis in ellagic acid treated cancer cells. Cancer Lett., 136: 215-221.

33. Moghimi, S.M., P. Symonds, J.C. Murray, A.C. Hunter, G. Debska and A. Szewczyk, 2005. A two-stage poly (ethylenimine)-mediated cytotoxicity: Implications for gene transfer/therapy. Mol. Ther., 11: 990-995.

34. Porter, A.G. and R.U. Janicke, 1999. Emerging roles of caspase-3 in apoptosis. Cell Death Differ., 6: 99-104.

35. Duhem, N., F. Danhier and V. Préat, 2014. Vitamin E-based nanomedicines for anti-cancer drug delivery. J. Control. Release, 182: 33-44. 